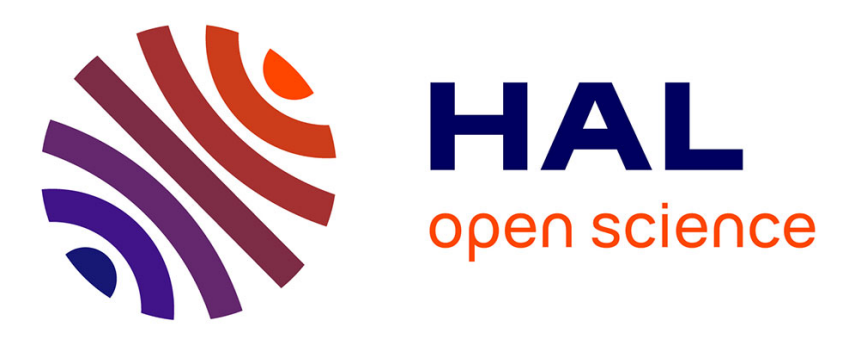

\title{
In-vitro study of pharyngeal pressure losses at the origin of obstructive sleep apnea
}

Annemie van Hirtum, Franz Chouly, A. Teulé, Yohan Payan, Xavier Pelorson

\section{To cite this version:}

Annemie van Hirtum, Franz Chouly, A. Teulé, Yohan Payan, Xavier Pelorson. In-vitro study of pharyngeal pressure losses at the origin of obstructive sleep apnea. 25th Annual Intemational Conference of the IEEE EMBS, Sep 2003, Cancun, Mexico. pp.371-374, 10.1109/IEMBS.2003.1279665 . hal-00108988

\section{HAL Id: hal-00108988 \\ https://hal.science/hal-00108988}

Submitted on 13 Aug 2020

HAL is a multi-disciplinary open access archive for the deposit and dissemination of scientific research documents, whether they are published or not. The documents may come from teaching and research institutions in France or abroad, or from public or private research centers.
L'archive ouverte pluridisciplinaire HAL, est destinée au dépôt et à la diffusion de documents scientifiques de niveau recherche, publiés ou non, émanant des établissements d'enseignement et de recherche français ou étrangers, des laboratoires publics ou privés. 


\title{
In-vitro study of pharyngeal pressure losses at the origin of obstructive sleep apnea
}

\author{
A. Van Hirtum ${ }^{1}$, F. Chouly ${ }^{2}$, A. Teulé ${ }^{1}$, Y. Payan ${ }^{2}$, and X. Pelorson ${ }^{1}$ \\ ${ }^{1}$ Institut de la Communication Parlée, UMR CNRS 5009, INPG-Université Stendhal, Grenoble, France \\ ${ }^{2}$ Laboratoire TIMC, faculté de médecine, Grenoble, France
}

\begin{abstract}
This paper presents an in-vitro experimental and theoretical study of pharyngeal airflow at the origin of obstructive sleep apnea. The air flow rate and pressure along a geometrical constriction are predicted from the upstream pressure and geometry. Quantitative results are validated for rigid 'in-vitro' replicas. The influence of asymmetry is discussed.
\end{abstract}

\section{INTRODUCTION}

Obstructive sleep apnea (OSA) is defined as the intermittent cessation of breathing during sleep. OSA is extensively shown to be an important health care issue with a reported prevalence of $4 \%$ in adult men and $2 \%$ in adult woman [1]. OSA causes excessive daily sleepiness and increases the development of cardiovascular diseases and arterial hypertension [2]. Consequently OSA has adverse consequences on the patients daily life and is associated with an increased risk on public traffic accidents [3]. Current research aim to improve diagnosis, follow-up and treatment and stresses the need for further understanding of the OSA syndrome in order to favour succesfull development of therapeutical and surgical treatments [4]-[7].

The presented work aims to contribute toward the complex interaction of the myriad factors determining pharyngeal airway obstruction. The OSA syndrome is known to be due to a partial (hypopnea) or total (apnea) collapse of the pharyngeal airway during inspiration [8]. From a physical point of view airway collapse is due to the fluid-mechanical interaction of the fluid (airflow during inspiration) and the surrounding structure (tissue). Studies of the biomechanical pharyngeal airflow and resulting forces in case of OSA are very limited. In [9] a polynomial function is proposed to predict the upperairway pressure-flow relationship as an objective measure of inspiratory flow limitation (IFL). Three-dimensional computational modelling of the airflow on a rigid human pharynx geometry is assessed in [10]. The pressure drop in the pharynx is quantified to lie in the range of $200-500 \mathrm{~Pa}$. The onset from laminar to turbulence flow is found to increase the presence drop with $40 \%$ and the subtle effects of OSA treatment on the airway morphology have a large effect on the pressure drop. This paper presents the results of an in-vitro experimental and theoretical study of pharyngeal airflow at the origin of the OSA syndrome. The air flow rate and pressure along a geometrical constriction are predicted from the supra-glottal pressure and geometrical information. The quantitative results are validated on rigid 'in-vitro' replicas.

\section{MATERIAL AND METHODS}

\section{A. In-vitro pharyngeal replica}

The place of obstruction of the pharynx at the origin of OSA is known to be very variable [7]. Regardless the precise location of obstruction in the pharynx (naso-, oro- or laryngopharynx) the relevant anatomy is 'in-vitro' imitated by a rigid demi-cylinder representing the tongue geometry placed inside a rectangular uniform pipe representing the pharyngeal wall. Changing the minimum aperture $\left(h_{\min }\right)$ between the tongue-replica and the pipe allows the study of different anatomical conditions. Consequently the important geometrical parameters are the diameter $\mathrm{D}$ of the demi-circle and the value of $h_{\min }$. In this study the diameter D of the rigid replica is fixed to $49 \mathrm{~mm}$ which is in accordance with anatomical 'in-vivo' values. Different degrees of constriction are studied by changing $h_{\min }$ between the demi-cylinder and the flat plate. Minimum distances $h_{\min }$ of $0.70,1.00,1.45$, 1.90 and $2.30 \mathrm{~mm}$ are considered. In order to connect the replica with the experimental set-up described in subsection II$B$ an attachment of length $25 \mathrm{~mm}$ and heigth $6 \mathrm{~mm}$ is fasten to the upper part of the demi cylinder maintaining a fixed vertical height of $34 \mathrm{~mm}$ at the origin i.e. between the beginning of the attachment and the flat plate. A longitudinal cross-section of the resulting pharyngeal geometry constituted from the attachment and 'in-vitro' tongue replica is depicted in Fig. 1 for the assessed $h_{\min }$ 's. Remark the strongly asymmetrical nature of the replicas geometry.

\section{B. Experimental set-up}

To simulate the origin of OSA the rigid pharyngeal replica is attached to an 'in-vitro' test-installation. The test-installation enables to study the influence of various incoming (inspiration) pharyngeal airflow conditions. To validate theoretical flow simulations, flow characteristics are measured at different positions along and upstream of the pharyngeal replica. Incoming airflow conditions are determined by measuring the inflow air rate $(\mathrm{q})$ and upstream pressure $\left(\mathrm{p}_{0}\right)$ as indicated in Fig. 1 where the direction of incoming airflow is indicated with an arrow. The inflow air rate $\mathrm{q}[1 / \mathrm{min}]$ is measured with a thermal mass flow meter (TSI 4040). Flow pressure measurements [Pa] are performed at three different sites $\left(\mathrm{p}_{1}, \mathrm{p}_{2}, \mathrm{p}_{3}\right)$ depicted in Fig. 1 along the converting part of the rigid tongue replica. The pressure is measured with piezoresistive pressure transducers (Endevco 8507C, Kulite XCS-093) positioned in pressure 


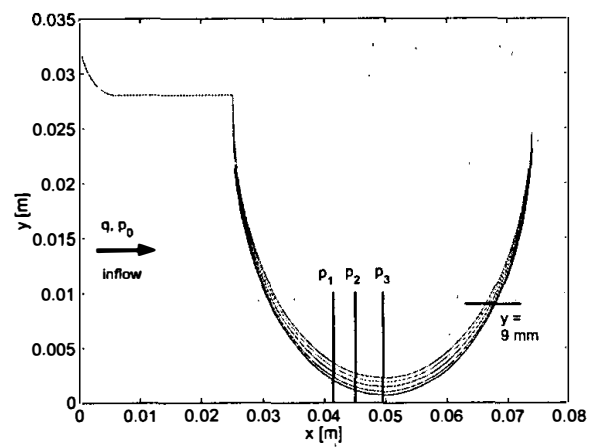

Fig. 1. Pharyngeal geometry represented by a flat bottom plate corresponding to $\mathrm{y}=0$ and demi-cylinders with diameter $\mathrm{d}=49 \mathrm{~mm}$ for the assessed $h_{\min }$ 's $(0.70,1.00,1.45,1.90$ and $2.30 \mathrm{~mm})$. The $y$-axis corresponds with the distance between the flat plate and the demi-cylinder. The $x$-axis presents the distance along the longitudinal axis of the replica. The sensor sites are indicated with a solid blue line.

taps of $0.4 \mathrm{~mm}$ diameter at the mentioned sites which allows dynamic pressure measurements. The site $\mathrm{p}_{3}$ corresponds to the position $h_{\min }$. The sites $\mathrm{p}_{2}$ and $\mathrm{p}_{1}$ are respectively located downstream from the site $\mathrm{p}_{3}$ at $4.5 \mathrm{~mm}$ and $8.0 \mathrm{~mm}$ along the $\mathrm{x}$-dimension. The air flow rate and pressure distribution $\mathrm{p}(\mathrm{x})$ along the replica are predicted from the measured inflow pressure $p_{0} . p(x)$ reveals the fluid-wall interactions of interest. The modelling performance of different flow models, described in II-C, towards measured experimental data is assessed. Next to pressure measurements a constant temperature anemometer system (IFA 300) is available in the test-installation to perform flow velocity measurements. Vertical velocity profiles along the $y$-dimension are measured with a spatial resolution of $\Delta z=0.1 \mathrm{~mm}$ at an opening of $9 \mathrm{~mm}$ indicated by a horizontal line in Fig. 1. A horizontal velocity profile is captured with a fixed x-position and steps $\Delta z$ of $0.1 \mathrm{~mm}$ and $1.0 \mathrm{~mm}$ in the $\mathrm{z}$-direction which is perpendicular to the $(\mathrm{x}, \mathrm{y})$ plane of Fig. 1 .

\section{Theoretical flow models}

The origin of OSA lies in a strong interaction of the fluid and the surrounding tissue. A first requirement to describe ongoing phenomena is to know the pressure variations through the pharyngeal geometry. Since an exact analytical solution for the flow through such a constriction is not available different flow models and flow assumptions are assessed to estimate the pressure $\mathrm{p}(\mathrm{x})$ as function of position [11]. Once $\mathrm{p}(\mathrm{x})$ is known the force $\mathrm{F}(\mathrm{x})$ acting by the airflow on the surrounding tissue of the pharynx is deduced as $F(x)=\int p d S$.

In a first approximation the flow is assumed to be 1) incompressible, 2) quasi-steady and 3) inviscid [12]. The assumption of incompressible flow is justified since the velocities achieved are small compared with the speed of sound in air which corresponds to a low Mach number. The velocities associated with respiration are typically not $>20 \mathrm{~m} / \mathrm{s}$, whereas the speed of sound is $\sim 350 \mathrm{~m} / \mathrm{s}$. The airflow can be considered as primarily steady as long as the flow pattems at any time are approximately the same, which is reasonable during quiet breathing at normal respiratory frequencies and rigid walls characterised by low Strouhal numbers. The neglection of vis- cous effects is motivated by considering the involved Reynold numbers of order $10^{3}$ which represents the ratio of inertial forces to viscous forces acting on a given fluid element and the length of the pharyngeal replica. These 3 assumptions allow to apply the steady one-dimensional (1D) Bernoulli law (1)

$$
p(x)+\frac{1}{2} \rho v(x)^{2}=c t e
$$

to estimate the pressure distribution along the pharyngeal walls. The volume flow velocity is defined by $\phi(x)=v(x) A(x)=$ cte with $\mathrm{v}(\mathrm{x})$ the local flow velocity and $\mathrm{A}(\mathrm{x})$ the area along the pharyngeal replica. So the pressure drop is due to changes in kinetic energy. Further an empirical ad-hoc correction is added to the 1-D Bemoulli equation to account for the occurrence of flow separation downstream of $h_{\text {min }}$. The jet formation downstream of the point of flow separation is due to very strong viscous pressure losses and reversed flow occuring near the wall. For a steady flow the onset of separation coinciding with the separation point is defined as $\left.\frac{\partial v}{\partial y}\right|_{y=0}=0$. In literature the area associated with flow separation $A_{s}$ empirically equals 1.2 times the minimum area $A_{\min }$ along the replica, i.e. $A_{s}=c A_{\min }$, with $c=1.2$ [13]. The ad-hoc correction for the $1 \mathrm{D}$ Bernoulli (1) results in a steady $1 \mathrm{D}$ expression for $\mathrm{p}(\mathrm{x})$, with $p_{0}$ and $A_{0}$ respectively the pressure and area upstream of the replica indicated in Fig. 1. The volume flow velocity is estimated as (3).

$$
\begin{gathered}
p(x)=p_{0}+\frac{1}{2} \rho \phi^{2}\left(\frac{1}{A_{0}^{2}}-\frac{1}{A(x)^{2}}\right) \\
\phi=A_{s} \sqrt{\frac{2\left(p_{0}\right)}{\rho}}, A_{s}=c A_{\min }
\end{gathered}
$$

The preceding assumption of inviscid flow is not valid for low Reynolds numbers. This is the case for low flow velocities or/and small $h_{\min }$ values. In this case an extra Poiseuille term is added to the Bernoulli expression for $p(x)$ in (2) to correct for viscous pressure losses. The Bermoulli expression with Poiseuille correction is given in (4) with $\mu$ the dynamic viscosity coefficient, $\mathrm{D}$ the diameter of the demi-cylinder and $h(x)$ the heigth between the demi-cylinder and the flat plate.

$$
p(x)=p_{0}+\frac{1}{2} \rho \phi^{2}\left(\frac{1}{A_{10}^{2}}-\frac{1}{A(x)^{2}}\right)-\frac{12 \mu \phi}{D} \int \frac{d x}{h(x)^{3}}
$$

In the preceding approaches the viscosity is either neglected (Bemoulli in (2)) or corrected with an additional Poiseuille term assuming a fully developed Poiseuille flow (Poiseuille (4)). In the Bemoulli case the velocity profile is assumed to be plane while in the Poiseuille case the profile is assumed to be purely parabolic. Although firstly the velocity at the walls is zero, which is not accounted for in the Bemoulli case, and secondly a fully Poiseuille flow isn't developed considering the dimensions of the pharyngeal replica (diameter D and heigth $\mathrm{h}$ ). Therefore it is likely to assume that the region in which viscous forces are important is confined to a thin layer adjacent to the wall which is referred to as laminar boundary layer $\delta$. The inviscid main flow, with velocity $U$, is described 
by Bernoulli (3). The resulting boundary layer theory is described by the Von Karnan momentum integral equation for steady flows [14]. An approximated method to solve this equation for laminar incompressible two-dimensional $(x, y)$ boundary layers is given by Thwaites method. Introducing two shape parameters $H(\lambda)=\frac{\delta^{*}}{\theta}, S(\lambda) \propto \frac{\tau_{S} \theta}{U}$ which are only functions of the velocity profile determined by the acceleration parameter $\lambda \propto \frac{d U}{d x} \theta$, with $\tau_{S}(x) \propto \lim _{y \rightarrow 0} \frac{\partial v}{\partial y}$ the wall shear stress indicating the viscous force per unit area acting at the wall, the displacement thickness $\delta^{*}$

$$
\delta^{*}(x)=\int_{0}^{\infty}\left(1-\frac{v(y)}{U}\right) d y
$$

and the momentum thickness $\theta$

$$
\theta(x)=\int_{0}^{\infty} \frac{v(y)}{U}\left(1-\frac{v(y)}{U}\right) d y .
$$

The Von Karman equation is then approximated by

$$
\theta^{2}(x) U^{6}(x)-\theta^{2}(0) U^{6}(0) \propto \int_{0}^{x} U^{5}(x) d x .
$$

Equation (7) in combination with the fitted formulas for $\mathrm{H}(\lambda)$ and $S(\lambda)$ tabelled in [11] enables to compute $U(x), p(x), \theta(x)$ and $\tau_{S}(x)$ up to separation where $\tau_{S}=0$. Moreover the point of separation $x_{S}$ is numerically estimated since separation is accepted to occur at $\lambda\left(x_{S}\right)=-0.0992$ [15]. So no ad-hoc assumption is made to account for flow separation.

The model performance is statistically quantified by the coefficient of determination $R^{2}=1-\frac{\hat{\sigma}^{2}}{\sigma_{y}^{2}}$ with $\hat{\sigma}^{2}$ the sample variance of the model residuals and $\sigma_{y}^{2}$ the sample variance of the measured system output about its mean value.

\section{RESULTS AND DISCUSSION}

The 3 steady flow models presented in II-C are 1D (Bemoulli, Poiseuille) or quasi-2D (boundary layer approximation) flow models. The z-dimension perpendicular to the $(x, y)$ plane is assumed to have no influence on the flow. In order to validate this assumption, the horizontal velocity profile is measured for each $h_{\min }$. If a flat profile is registered the assumption is confirmed. Fig. 2 illustrates an exemplary velocity profile for $h_{\min }=2.3 \mathrm{~mm}$ and a steady flow of $601 / \mathrm{min}$. The step $\Delta z$ at the edges near the wall obtains $0.1 \mathrm{~mm}$ elsewhere $\Delta z$ equals $1.00 \mathrm{~mm}$. The anemometer is positioned as close as possible to the minimum aperture. The measured velocity including the edges yields $12.2 \mathrm{~m} / \mathrm{s}$ on average with a standard deviation $(\xi[\%]$ ) of $0.08 \mathrm{~m} / \mathrm{s}$ (or $\xi<1 \%$ ). $\xi<1 \%$ corresponds to a flat velocity profile along the z-direction. For all assessed apertures $\xi<1 \%$ and neglecting the $z$-dimension in the flow description is motivated. Moreover notice the good agreement between the measured velocity of $12.2 \mathrm{~m} / \mathrm{s}$ and the theoretical velocity of $12.9 \mathrm{~m} / \mathrm{s}$ for the case depicted in Fig. 2 .

Pressure measurements at $p_{1}, p_{2}$ and $p_{3}$ and Bernoulli simulations normalised with the upstream inflow pressure $p_{0}$ are presented in Fig. 3(a). $h_{\min }$ is set to $1.0 \mathrm{~mm}$ and the

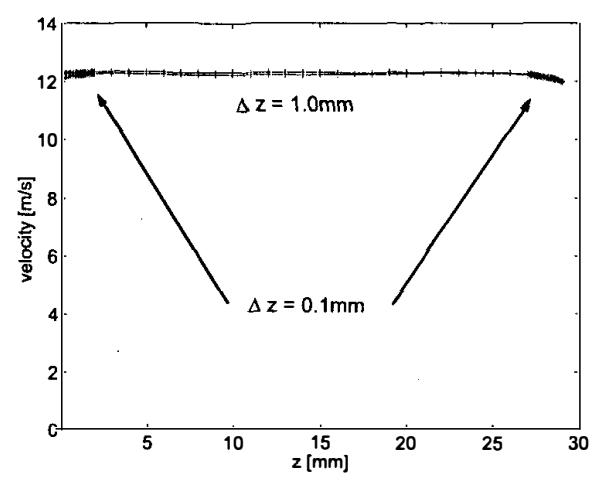

Fig. 2. Horizontal velocity profile ( 0 up to $30 \mathrm{~mm}$ and 30 down to $0 \mathrm{~mm}$ ) for $h_{m}$ in $=2.3 \mathrm{~mm}$ and a steady flow of $60 \mathrm{~V} / \mathrm{min}$.

inflow rate q varies from 5 up 1201/min in steps of 51/min. Fig. 3(a) shows the Bemoulli curve for two different positions of flow seperation depending on the value of the constant $\mathrm{c}$. The full line curve on top is obtained for the value retrieved in literature $c=1.2$ while the dotted curve on bottom corresponds with $c=1.05$. The value $c=1.05$ is retrieved from the measured data as $c=\sqrt{1-\frac{p_{3}}{p_{0}}}=1.05$. Fig. 3(a) illustrates the impact of the 'ad-hoc' value $c$ or the position of flow separation on the flow. The origin of the OSA is qualitatively explained by the negative pressure at the level of the constriction. An accurate quantitative model is obtained for the region of maximal pressure drop $\left(\mathrm{R}^{2}=0.97\right.$ at site $\left.p_{3}\right)$ with a simplified 1D Bemoulli flow description. The value $c$ is altered in comparison with the value reported in literature due to the asymmetrical geometry. For small constiction heights (small $h_{\min }$ at site $p_{3}$ ) and small flow rates $q$ the Poiseuille correction term for viscous pressure losses in (2) might becomes important. Although the influence is limited as seen from the small change in model performance at site $p_{3}, \mathrm{R}^{2}=0.98$ instead of $\mathrm{R}^{2}=0.97$ in the Bernoulli case. Fig. 3(b) illustrates the measured vertical velocity along the $y$ dimension for a fixed $x$-value indicated by a horizontal line in Fig. 1. The inflow rate $\mathrm{q}$ ranges from $201 / \mathrm{min}$ up to $1001 / \mathrm{min}$, $h_{\min }=2.30 \mathrm{~mm}$ and $\mathrm{y}=0$ corresponds with the flat plate of the replica. The plotted profiles show the existence of a boundary layer near the edge $\left(y / h_{\min }=0\right)$ and the formation of a jet since the velocity tends to 0 after $y / h_{\min }=1$. Moreover for high flow rates $\mathrm{q}$ the profile shows asymmetric shoulders indicated by arrows due to the asymmetry of the replica. Thwaites approximated solution of the boundary layer equation allows to predict the pressure and point of separation as exemplary illustrated for $h_{\min }=1.00 \mathrm{~mm}$ in respectively part a) and part b) of Fig. 3(c). Consequently the ad-hoc value $\mathrm{c}=1.05$ applied to optimise the $1 \mathrm{D}$ modeling performance is an unphysical value.

The overall model performance at all measuring sites $\left(p_{1}, p_{2}, p_{3}\right)$ for Bernoulli, Poiseuille and Thwaites for all values of $h_{\min }(0.7,1.0,1.4,1.9,2.3 \mathrm{~mm})$ is summarised in Table I. The model accuracy is expressed by the coefficient of determination $R^{2}$. The significant loss in accuracy at the positions $\mathrm{p}_{1}$ and $\mathrm{p}_{2}$ is due to several reasons. As illustrated in 


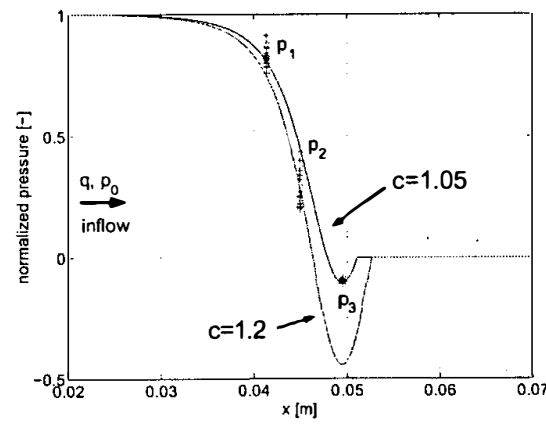

(a) Bernoulli flow simulation.

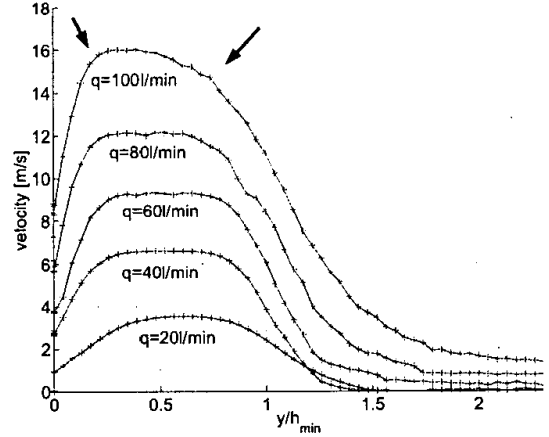

(b) Measured velocity profile at $\mathrm{y}=9 \mathrm{~mm}$.
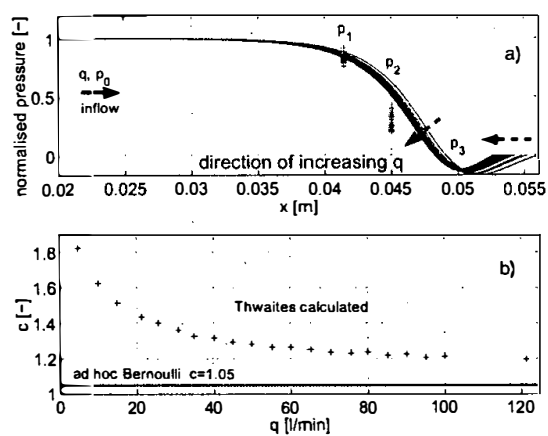

(c) Thwaites flow simulation.

Fig. 3. (a) pressure measurements at $p_{1}, p_{2}$ and $p_{3}(+)$ and Bernoulli simulations with $\mathrm{c}=1.05$ (full line on top) and $\mathrm{c}=1.2$ (dotted line below) normalised with the inflow pressure $p_{0}$ for $h_{\min }=1.00 \mathrm{~mm}$ and $\mathrm{q}=51 / \mathrm{min}$ up to $\mathrm{q}=1201 / \mathrm{min}$. (b) measured vertical velocity profile at $\mathrm{y}=9 \mathrm{~mm}$ for given flow rates $\mathrm{q}$. (c) a) pressure measurements at $p_{1}, p_{2}$ and $p_{3}(+)$ and Thwaites approximations (full lines) normalised with the inflow pressure $p_{0}$ for $h_{m i n}=1.00 \mathrm{~mm}$ and $\mathrm{q}=51 / \mathrm{min}$ up to $\mathrm{q}=1201 / \mathrm{min}$. b) $\mathrm{c}$ as function of $\mathrm{q}(+)$ from Thwaites calculations and the ad hoc $\mathrm{c}=1.05$ (full line) used in Bernoulli flow simulations.

TABLE I

FLUID MECHANICAL MODEL PERFORMANCE

\begin{tabular}{|c|c|c|c|}
\hline & $\mathrm{R}^{2}$ for $\mathrm{p}_{1}$ & $\mathrm{R}^{2}$ for $\mathrm{p}_{2}$ & $\mathrm{R}^{2}$ for $\mathrm{p}_{3}$ \\
\hline Bernoulli & 0.54 & 0.48 & 0.97 \\
Poiseuille & 0.54 & 0.45 & 0.98 \\
Thwaites & 0.53 & 0.37 & 0.94 \\
Thwaites(asym) & 0.76 & 0.65 & 0.87 \\
\hline
\end{tabular}

Fig. 3(a) and part a) of 3(c) the steepness of the pressure slope at the sites $\mathrm{p}_{1}$ and $\mathrm{p}_{2}$ will increase the sensitivity to the exact sensor position. Secondly the asymmetry of the replica (curved cylinder versus flat plate) lowers the accuracy towards the site $\mathrm{p}_{3}$. To compensate for the asymmetry the Thwaites simulation is repeated for a demi-cylinder with doubled diameter, so $2^{*} \mathrm{D}$ instead of $\mathrm{D}$. The modeling performance is given in the last row of Table I labelled Thwaites(asym). $\mathrm{R}^{2}$ is significantly increased for Thwaites(asym) compared to Thwaites at the sites $p_{1}$ and $p_{2}$. The obtained pressure estimations are included in a finite element model of the tongue.

\section{CONCLUSION}

The theoretical and 'in-vitro' study of the pharyngeal pressure drop at the origin of the OSA syndrome is quantitatively modelled $R^{2}>0.9$ at the position of minimum aperture in the pharyngeal replica. The adverse influence of the asymmetry on the modelling appears in the unphysical position of the flow separation upstream of the constriction in case of the $1 \mathrm{D}$ approaches and in the reduced model performance along the slope of the replica. So a simple $1 \mathrm{D}$ approach results in the strived pressure values, but it should be kept in mind that an unphysical position of the flow separation upstream of the constriction is applied. A small variation in geometry is likely to alter the optimal 1D position of separation. Therefore although computational favourable, application of more advanced fluid models is appropriate. The quasi-2D Thwaites solution of the boundary layer equation results in a physical meaningful solution. Further research towards the asymmetry is likely to improve the overall model performance.

\section{ACKNOWLEDGMENT}

This work is funded by the Rhone-Alpes region, France.

\section{REFERENCES}

[1] T. Young, M. Palta, J. Dempsey, J. Skatrud, S. Weber, and S. Badr, "The occurrence of sleep-disordered breathing among middle-aged adults," New England J. of Medicine, vol. 17, no. 328, pp. 1230-1235, 1993.

[2] P. Peppard, T. Young, M. 'Palta, and J. Skatrud, "Prospective study of the association between sleep-disordered breathing and hypertension," New England J. of Medicine, vol. 342, no. 19, pp. 1378-1374, 2000.

[3] J. Teran-Santos, A. Jimirez-Gomez, and J. Cordero-Guevara, "The association between sleep apnea and the risk of traffic accidents," New England J. of Medicine, vol. 340, no. 11, pp. 847-851, 1999.

[4] D. Hui, D. Choy, F. Ko, T. Li, and C. Lai, "Osa syndrome : reatment update," Medical practice, vol. 6, no. 2, pp. 209-217, 2000.

[5] W. McNicholas, "Sleep apnoea syndrome today: much done, more to do," Sleep Medicine Reviews, vol. 7, no. 1, pp. 1087-1093, 2003.

[6] T. Penzel, J. McNames, P. de Chazal, B. Raymond, A. Murray, and G. Moody, "Systematic comparison of different algorithms for apnoea detection based on electrocardiogram recordings," Medical \& Biological Engineering \& Computing, vol. 40.

[7] A. Rama, S. Tekwant, and C. Kushida, "Sites of obstruction in obstructive sleep apnea," Chest, vol. 122, no. 4, pp. 1139-1147, 2002.

[8] I. Ayappa and D. Rapoport,."The upper airway in sleep: physiology of the pharynx," Sleep Medicine Reviews, vol. 7, no. 1, pp. 9-33, 2003.

[9] K. Mansour, J. Rowley, A. Meshenish, M. Shkoukani, and M. Badr, "A mathematical model to detect inspiratory flow limitation during sleep," Medical \& Biological Engineering \& Computing, vol. 93.

[10] B. Shome, L. Wang, M. Santare, A. Prasad, A. Szeri, and D. Roberts, "Modeling of airflow in the pharynx with application to sleep apnea," J. of biomechanical engine ering, vol. 120.

[11] R. Blevins, Applied Fluid dynamics handbook. Malabar: Krieger publishing company, 1992.

[12] A. Fishman, P. Macklem, J. Mead, and S. Geiger, The respiratory system. In Handbook of physiology. Maryland: Am. Phys. Soc., 1986.

[13] Y. Payan, M. Chabanas, X. Pelorson, C. Vilain, P. Levy, V. Luboz, and P. Perrier, "Biomechanical models to simulate consequences of maxillofacial surgery," C. R. Biologies, vol. 325.

[14] H. Schlichting and K. Gersten, Boundary layer theory. Berlin: Springer Verlag, 2000.

[15] X. Pelorson, A. Hirschberg, R. V. Hasselt, A. Wijnands, and Y. Auregan, "Theoretical and experimental study of quasisteady-flow separation within the glottis during phonation. application to a modified two-mass model." $J A S A$, vol. 96, no. 6, pp. 3416-3431, 1994. 